\title{
ENSAYOS
}




\section{EL TRABAJO INFANTIL EN AMÉRICA LATINA}

\section{Maria Cristina Salazar}

En América Latina y el Caribe, a partir de la ratificación de la Convención de la ONU de 1989, ocurre una saludable proliferación de estudios sobre el trabajo infantil y juvenil, y comienza a surgir una conciencia pública mayor sobre los efectos - a veces perversosdel ingreso prematuro de millones de niños y niñas en el mercado laboral y, en particular, de su participación en trabajos dañinos o peligrosos que afectan su desarrollo, salud y escolaridad. ${ }^{1}$ Tanto los gobiernos como la sociedad civil han comenzado a responder a este problema, mediante la elaboración de nuevas políticas y programas sociales, inspirados muchos de ellos en el espíritu del artículo 32 de dicha Convención ${ }^{2}$.

\section{Distribución y extensión del trabajo infantil}

Un resumen rápido de este problema necesariamente debe destacar su magnitud. Estimaciones de la OIT indican que en la región hay varios millones de niños, niñas y adolescentes trabajando en condiciones heterogéneas; alrededor de 7 millones son menores de 10 a 14 años. Como muchos niños que trabajan tienen menos de 10 años, podría estimarse que el total de niños y niñas trabajadores oscilada de 15 a 20 millones. Muchos se encuentran en las calles de las grandes ciudades como vendedores callejeros prematuros, otros trabajan en talleres clandestinos, unos pocos (casi siempre mayores de 14 años) en empresas formalmente constituidas, mientras que un segmento mayoritario se encuentra desarrollando labores agrícolas, en las que niños y niñas participan desde los 5 o 6 años. Casi todos ellos se vinculan al sector informal de la economía, sin contrato y sin segundad social. Es más probable disponer de un contrato en la ciudad que en el campo. La desprotección tiende a ser mayor entre los niños de menor edad. Cientos de miles de niñas y adolescentes se concentran en los servicios, en particular en el servicio doméstico. Niños, niñas y adolescentes trabajan, siendo invisibles para la sociedad, el Estado y los sindicatos. Casi todos ellos desconocen sus propios derechos.

En Guatemala, por ejemplo, la participación laboral de niños y niñas de 7 a 12 años significa el $27 \%$ del total del trabajo de menores de edad en ese país. En Ecuador, el $12 \%$ de la población trabajadora infantil tiene entre 10 y 11 años de edad. En Perú, el trabajo involucra al $16 \%$ de los niños y adolescentes que se hallan entre los 6 y los 17 años de edad. En Colombia, el trabajo de los menores de 12 años es un problema de grandes dimensiones, en especial en las zonas rurales, donde 1 de cada 3 niños de 10 a 11 años y 1 de cada 4 de 6 a 9 años trabajan en actividades secundarias. ${ }^{3}$ En las grandes ciuda-

\footnotetext{
${ }^{1}$ Esta ponencia recoge algunos resultados de informes preparados dentro del programa mundial del International Working Group on child Labour (Brasil, Colombia, Chile y Guatemala) (véase Salazar y Gárate, 1996) y de estudios de caso sobre la relación entre el trabajo infanto-juvenil y la educación realizados para UNICEF, Mejores escuelas: menos trabajo infantil. Estudios de caso sobre educación y trabajo infantil en Brasil, Colombia, Ecuador, Guatemala y Perú. Editores: W. Alarcón y M.C. Salazar. 1996. Unicef América Latina. Santafé de Bogotá. Los estudios de caso aparecen en la bibliografía bajo Alarcón, García, Rodríguez, Rizzini et al., Turbay y Acuña.

${ }^{2}$ Dicho artículo reza así: "Los Estados Partes reconocen el derecho del niño a estar protegido contra la explotación económica y contra el desempeño de cualquier trabajo que pueda ser peligroso o entorpecer su educación, o que sea nocivo para su salud o para su desarrollo físico, mental, espiritual, moral o social."

${ }^{3}$ Las actividades secundarias sólo se miden en el área rural y consisten en cuidar animales, trabajar en una huerta casera, hacer mejoras y ayudar en una tienda o negocio, sin tener en cuenta el número de horas trabajadas.
} 
des, como lo señalan Flórez ti al. (1995: 108), el problema no deja de ser significativo: 1 de cada 6 niños de 10 a 11 años, y 1 de cada 10 de 6 a 9 años, participan en el mercado laboral.

La rama de actividad económica que genera mayores tasas de participación laboral de los niños es la agricultura. En algunos casos se trata de labores agrícolas tradicionales, derivadas de formas de producción que sólo permiten la subsistencia del núcleo familiar. En otras ocasiones, la ocupación de los niños se inserta en cultivos orientados al mercado, e incluso a la exportación. En Guatemala se encuentra que el $65 \%$ del total de la población trabajadora menor de edad se ocupa en la agricultura, siendo mayor el porcentaje para los niños indígenas que para los no indígenas; en Ecuador y en Perú, el $48 \%$ y el $40 \%$ respectivamente de la población de niños y adolescentes que trabajan, lo hacen en actividades agropecuarias. La misma tendencia se constata en los informes de Brasil (78.5\% de los niños trabajadores de 10-17 años lo hacen en la agricultura) y Colombia (donde el $82.6 \%$ de los varones y el $36.0 \%$ de las niñas de 10 a 17 años que trabajan se desempeñan en la rama de actividad agropecuaria).

Hay muy pocos datos de niños trabajadores de las distintas etnias, a pesar de tratarse de países pluriétnicos; para Guatemala, Ecuador y Perú, se presentan algunos datos al respecto que indican una mayor explotación económica para los niños y niñas indígenas. Se requiere mayor información sobre los núcleos familiares y la vida y el trabajo de los niños en las distintas ocupaciones y en la calle, aunque se constata cada vez más su vínculo con condiciones de pobreza extrema.

Desde otro ángulo, el género es una variable que juega un papel importante: son más los varones que las niñas que trabajan en todos los países. La información de Guatemala reporta que para el tramo de la población que trabaja entre 7 a 17 años, 84\% son varones y $16 \%$ mujeres. La estadística de población entre 10 a 17 años que trabaja en Ecuador es de $64 \%$ para varones y $36 \%$ para mujeres. En el caso de Brasil, para el tramo de edad entre 10 a 14 años tenemos un 63\% de varones frente a $37 \%$ de mujeres. La misma tendencia se corrobora con la información de Colombia y Perú: el $70 \%$ de los adolescentes colombianos (12 a 17 años) que trabajan son varones. En Perú para el grupo de trabajadores entre 6 a 17 años el $60 \%$ igualmente son varones.

Sin embargo, aquí es necesario señalar que frecuentemente la participación en particular de niñas y adolescentes mujeres adolece de un significativo sub-registro en la conceptualización tradicional de la población económicamente activa, ya que no se consigna como trabajo la participación de éstas en actividades domésticas en su propio hogar, aunque en muchos casos estas tareas suponen el abandono de la escuela y jornadas laborales excesivas. Un segmento no despreciable de niñas y mujeres adolescentes tiene una doble jornada laboral. Salen a trabajar fuera de casa, pero además cumplen con tareas domésticas al regresar a su propio hogar En Guatemala, por ejemplo, si a la jornada de trabajo de 40 horas que en promedio realizan las niñas en dicho país se le añaden las 21 horas que dedican a ocupaciones caseras, se obtendría una jornada total de 61 horas de trabajo a la semana. Este es uno de los temas que merece mayor atención por parte de los analistas.

\section{Aportes económicos}

Los aportes económicos de estos niños al presupuesto familiar no son muy significativos: se calcula que suman alrededor de un $10 \%$ de ese presupuesto. Es tres veces mayor el 
aporte de los adolescentes (de 13 años en adelante) al presupuesto familiar, y ellos predominan dentro de la fuerza de trabajo de los menores de edad. A medida que transcurre la edad, un contingente mayor se incorpora al trabajo, siendo así que la mayor proporción de trabajadores menores de edad en nuestros países está localizada en el grupo de 15 a 17 años. No sabemos con precisión si este aporte monetario es vital o no para la subsistencia inmediata de las familias populares. Entre otras razones, porque los ingresos de los niños y adolescentes resultan tan bajos, que en la mayoría de los países representan sólo la mitad de lo percibido por los asalariados de 35 a 54 años de edad, con escasa escolaridad (7 años de estudio) (CEPAL, 1995).

En algunos casos, como en el sur del Brasil y en Chile, una gran proporción de los niños y adolescentes trabajadores destina los ingresos para sí mismos. Esto no significa que la carencia económica no sea el móvil principal para trabajar. La diferencia parece radicar en que -en estos casos-es más fuerte la interferencia de factores culturales, que impulsan a los niños a independizarse económicamente con mayor precocidad. Es también bastante común la entrega del dinero obtenido por parte de los niños a sus familias, sea en parte o totalmente. Pero no hay que olvidar que las proporciones de trabajadores infantiles y adolescentes que no tienen ninguna remuneración son bastante altas.

El trabajo de niños y adolescentes está categorizado mayoritariamente como "trabajo familiar no remunerado", siendo éste mayor en los niños que entre los adolescentes, y en zonas rurales más que en urbanas. En Ecuador, el $57 \%$ del total de la población trabajadora infanto-juvenil se clasifica como "trabajador familiar no remunerado", mientras que en el Perú el 44\% se ubica en esta misma categoría. En Colombia, el $44.2 \%$ del total de trabajadores de 12 a 13 años y el 26.8\% de los de 14 a 17 años, son trabajadores familiares sin pago (Flórez et al., 1995: Cuadro 8.1).

La CEPAL afirma que en la región es baja la incidencia del ingreso proveniente del trabajo infantil y adolescente en los niveles globales de indigencia y pobreza, pero a la vez es muy elevado en los estratos específicos de hogares con niños y adolescentes trabajadores, lo que alienta a impulsar programas que permitan aliviar las necesidades presentes de estos hogares para así postergar la incorporación al mercado laboral y permitir que acumulen más capital educacional en ese período (ibid.: 54-55).

Los estudios de este decenio avanzan en el reconocimiento de la heterogeneidad del trabajo infantil marcada por las desigualdades de las regiones y su desarrollo en cada país. Las áreas rurales presentan grandes diferencias y el trabajo infantil difiere según se trate de economías campesinas o de áreas de producción agroindustrial. El atraso de los niveles tecnológicos de muchas áreas rurales y la ausencia de reformas agrarias que afecten la distribución y uso de la tierra contribuyen a la mayor explotación económica de los niños trabajadores. Con frecuencia el trabajo infantil se realiza dentro del contexto de ayuda a la familia, sin protección alguna.

\section{Trabajo infantil y pobreza}

Se esclarece en las investigaciones la compleja relación entre trabajo infantil y pobreza. No podemos afirmar que el trabajo infantil sea factor determinante de la transmisión intergeneracional de la pobreza, pero no parecen existir dudas acerca de que la incorporación prematura al trabajo mantiene los niveles de pobreza en determinados estratos. Igualmente sabemos que esa vinculación laboral se debe principal, pero no exclusivamente, a la pobreza y que contribuye muchas veces a la pobreza futura de los 
niños y niñas trabajadores. Los estudios documentan la relación inversa que existe entre ingreso familiar y participación en el mercado de trabajo. Cuanto más bajo el ingreso per capita familiar, mayor la proporción de los que declaran trabajar para complementar el presupuesto familiar, aunque hay excepciones. Hay más familias pobres que niños trabajando y, si la pobreza familiar bastase para explicar el trabajo de los niños, cabria preguntarse las razones de por qué no hay más niños ocupados en actividades laborales. En otros términos, la pobreza es un factor necesario para la existencia del trabajo infantojuvenil, pero no es factor suficiente para la aparición y permanencia de este problema.

Inclusive, estadísticas en diversos países latinoamericanos encuentran que un segmento de niños y jóvenes trabajadores pertenecen a familias que no son pobres. Es decir, están adscritos a grupos familiares que están sobre la línea de la pobreza. En este sentido es interesante resaltar los datos que reporta el Perú: $30 \%$ de niños y adolescentes trabajadores pertenecen a familias "no pobres". Este dato expresa la realidad de familias empobrecidas de las clases medias quienes, no obstante haber logrado superar el límite de la pobreza, por sus condiciones de vida están más ligadas al extenso sector de los pobres que al reducido estrato pudiente de nuestras sociedades.

\section{Factores culturales}

Los niños trabajan, en general, porque su familia es pobre, pero también por factores culturales. La concepción que subyace en el fondo parece ser la de que todos los miembros de la familia son proveedores económicos de ella, y mediante el ejercicio de esta responsabilidad se forma a los niños de hoy para que sean los adultos competentes del mañana. En todos los países se encontraron formas de producción en las que la familia actúa como bloque, dándose por sobreentendida la colaboración activa de los niños. Los padres justifican la vinculación de sus hijos al trabajo aduciendo que en él adquieren valores como la responsabilidad, la autonomía y la tenacidad para sobrellevar las dificultades o para soportar sacrificios. Además se interpreta al trabajo como una protección contra los vicios y el ocio "que conduce a la delincuencia".

La percepción acerca de la escuela es ambivalente. Por una parte se valora la posibilidad de aprender a leer y escribir, pero por lo menos un sector de padres rurales percibe esta educación como irrelevante, y cuando los horarios de la escuela y del trabajo se contraponen, tienden a privilegiar el trabajo, por cuanto éste tiene beneficios inmediatos evidentes para la subsistencia de la familia; la asistencia a la escuela, en cambio, no. Es la disyuntiva entre la posibilidad de movilidad social futura que les abre la escuela y las urgencias de la sobrevivencia presente.

Muchos padres temen a la escuela y al estudio. "Yo aprendí así", es común que digan. Recoger su propia experiencia anterior como niños trabajadores para justificar el trabajo de sus hijos es uno de los mecanismos más utilizados.

En Brasil y en Colombia se señala que para un sector de padres la incorporación laboral de sus hijos tiene un objetivo formativo; es decir, pretenden que el trabajo sea un espacio de promoción de valores como la responsabilidad o la disciplina, o como un lugar de "preparación para la vida". Según esta perspectiva el trabajo operaría como una suerte de escuela para la vida. Existen, de otro lado, segmentos de familias populares para quienes el trabajo prematuro se convierte en mecanismo preventivo a la ociosidad. Esto tiene que ver con la escasa valoración que se da en ciertos sectores populares al juego de los niños. La actividad lúdica es vista por muchos como pérdida de tiempo, sin avizorar la importancia del juego en su formación. 
Los empleadores también piensan que al vincular los niños al trabajo precoz, ellos los están ayudando, lo que los lleva a pensar que no tienen por qué pagar justamente su trabajo. Estas formas de conceptualizar el trabajo como una manera de"salvar" al niño tienden a legitimar el trabajo infantil de los niños y adolescentes pobres. Pero esta clase de razonamiento dificulta las metas de erradicación progresiva al sacar la discusión del terreno de los derechos del ciudadano, ubicándola en el terreno ideológico o de la filantropía, ocultando la relación compra/venta del trabajo y la relación patrón/empleado (Rodrigues dos Santos, 1995).

Tienen acentuada presencia, en particular en zonas rurales, concepciones premodernas de la infancia, en las que niños y niñas son concebidos solamente como "adultos en miniatura". En estos casos los niños son recargados de actividades laborales, en tanto éstas forman parte de su función como tales.

La incidencia de variables demográficas asociadas a la temprana incorporación laboral de los niños es un aspecto que resaltan diversos estudios. Existe un relativo mayor empobrecimiento de los hogares dirigidos por mujeres solas, y en éstos es mayor la probabilidad de que los niños abandonen la escuela para convertirse en trabajadores. Para Guatemala se informa que los hogares de madres solas están asociados, no tanto al trabajo infantil, cuanto al abandono escolar de los niños. Aunque los factores de discriminación étnica también entran en juego, posiblemente estos niños que no estudian están concentrados en actividades domésticas o agrícolas en su propio hogar.

\section{Trabajos riesgosos}

Se han documentado mejor los trabajos riesgosos para los niños y los jóvenes, que tienden a ser los mismos en los países estudiados. La minería del carbón en Colombia y Chile; las quemas de madera para producir carbón vegetal en Brasil; la minería del oro en Colombia y Perú; las actividades agrícolas en las plantaciones de caña, cebolla, fique y tabaco, o en la agroindustria de las flores y frutas para la exportación, en las que se presentan accidentes por el uso de herramientas de difícil manejo como el machete, y donde los adolescentes distribuyen los productos agroquímicos sin ninguna protección adecuada, son algunos de los ejemplos analizados. La industria de fabricación de cohetes y juegos pirotécnicos es una de las que más emplea niños y adolescentes en Guatemala y en Colombia. En las ciudades, las ventas y otras actividades callejeras, entrañan peligros físicos para los niños, y además su acercamiento a negociantes ilegales o delincuentes comunes. Todas estas ocupaciones impiden la escolarización y el desarrollo de los niños, por lo que deben ser eliminadas para todos los menores de edad, como se proclama en las leyes, pero para cuyo cumplimiento no se proveen mecanismos de control y fiscalización eficientes por parte del Estado.

\section{Trabajo infantil y escolaridad}

Se ha documentado mucho más la relación entre trabajo infantil y escolaridad que tiende a ser excluyente, y afecta sobre todo a los estratos más pobres de la población. La deserción, la repitencia, la falta de rendimiento escolar, la extraedad y el atraso escolar caracterizan a los niños trabajadores. No puede ser de otra manera mientras subsistan condiciones laborales en las que se comprueba claramente que el trabajo infantil no tiene un carácter esporádico, pues en todos los países se señalan jornadas laborales que equivalen y aun superan a las de los adultos. 
Los estudios se refieren a la situación atrasada, muchas veces desastrosa de la educación; se habla de la "escuela fracasada" que en lugar de atraer a los niños y niñas, los expulsa. Se señala la urgencia de transformar el sistema educativo, aumentar la inversión estatal en educación, mejorar los niveles de los maestros e introducir pedagogías nuevas, para que la escuela se convierta en imán de los niños. Se enfatiza la necesidad de una voluntad política expresa y clara de retener a los niños en la escuela como el primer objetivo por lograr dentro de la meta de erradicación del trabajo infantil. Es necesario reincorporar a los niños que desertan por motivos económicos, y en particular por la ineficacia de la escuela, para vincularse al mercado de trabajo. Urge impulsar un proceso de apropiación colectiva del sistema escolar para convertirlo en un ámbito público, y desarrollar la capacidad social de plantear problemas y soluciones y generar responsabilidades compartidas.

Los niños que nunca asisten a la escuela siguen siendo numerosos. En Brasil, estudios de caso en Sáo Paulo y Recife, con niños callejeros trabajadores de 10 a 14 años, encontraron porcentajes altos entre los que nunca frecuentaron la escuela (56\% y $76 \%$ respectivamente). La carencia de un aprestamiento escolar incrementa las posibilidades de fracaso, repetición y deserción para los niños de los sectores más pobres. El mejoramiento de la educación primaria depende de una atención preescolar equitativa y suficiente (UNICEF, 1992a).

En Perú, solamente el 33\% del total de la población trabajadora entre 6 a 17 años asiste a algún centro educativo. En Brasil, se corrobora que, de los niños y adolescentes entre 10 a 17 años, aquellos que tienen mayor tasa de actividad poseen menor tasa de escolaridad. En Colombia se encuentra que del total de la población infanto-juvenil que trabaja, la proporción que puede combinar trabajo con estudio es siempre menor a aquella que solamente se dedica a trabajar. La proporción de trabajadores del grupo de 12 a 13 años que estudia es $29.6 \%$, frente al $89.5 \%$ de la misma edad que no trabajan pero si estudian. Se mantiene una diferencia similar en el grupo de 14 a 17 años: los trabajadores que estudian son el 20.3\%, mientras que entre los no trabajadores esta proporción aumenta al 80.4\% (Flórez et al. 1995: Cuadro AS. 1). La investigación de Ecuador reporta que el promedio de niños y adolescentes matriculados en un centro educativo es mucho mayor entre quienes no trabajan (89\%) que entre quienes trabajan (58\%).

Las evidencias en América Latina son nítidas al demostrar que la mayor parte de niños y adolescentes que trabajan, o un sector significativo de éstos, no asisten a la escuela. Este es un punto de central importancia. Ciertamente sería erróneo suponer que el trabajo es el único factor que impide la asistencia escolar. Además de la condición laboral existen variables diversas que inciden en la no incorporación o abandono del sistema escolar. No obstante, las evidencias que tenemos nos conducen a admitir que el trabajo prematuro es una variable que difícilmente es compatible con la asistencia escolar. Más bien se trata de una relación excluyente.

Este fenómeno muestra matices según edades y localización geográfica de los trabajadores prematuros. En este campo es necesario examinar el problema no exclusivamente por el lado de cómo el trabajo obstaculiza la asistencia escolar; sino también por el lado de cómo esta escuela no satisface las demandas y expectativas de los educandos, constituyéndose así en un factor de expulsión.

En el campo se muestra con mayor claridad el desencuentro entre la educación que se ofrece y las necesidades reales de las familias. Las prácticas culturales favorecen el trabajo de los niños desde temprana edad. En la población indígena de Guatemala se 
evidencia este fenómeno: el niño mantiene una estrecha relación con su madre y participa directa o indirectamente de todas sus actividades. Poco a poco se aumenta la exigencia de disciplina y autonomía a que sus padres lo exponen, y esta exigencia se manifiesta en la obligación que se le impone de asumir por si mismo tareas productivas y domésticas. El juego es visto como una pérdida de tiempo; los padres piensan que trabajando, los niños aprenden más.

Si se examina la educación con base en sus resultados, encontramos en general una situación crítica. Altas tasas de repetición y, consecuentemente, de atraso escolar. La deserción escolar mantiene niveles elevados. Es decir, ni siquiera en sus propios términos (independiente de la crítica a los contenidos o metodologías educativas) esta escuela es exitosa. Si esta es la situación global, los niños de las clases populares se ven particularmente desfavorecidos y, dentro de este segmento, aquellos que trabajan. Según se informa en el caso peruano, entre la población de 6 a 14 años el atraso escolar de los no trabajadores es de 39\%, mientras que el atraso escolar de los trabajadores alcanza el $61 \%$. De la misma manera, la deserción de los no trabajadores para el mismo tramo de edad es de $8 \%$, en tanto que el de aquellos que trabajan tiene una tasa de deserción de 47\%. En Brasil se encuentra que entre la población de 10 a 17 años, el 73\% de los que no trabajaban estaban atrasados en la escuela, en tanto que el $86 \%$ de los trabajadores estaban en la misma condición de atraso escolar. Las investigaciones de Colombia, Guatemala y Ecuador coinciden en vincular el trabajo prematuro al atraso y abandono de la escuela.

\section{Los sistemas educativos}

La primaria. Se conoce la alta correlación entre la inversión nacional en educación primaria y el crecimiento económico. A pesar de que los gobiernos reconocen esta relación, no se ha prestado suficiente atención a la necesidad de aumentar el gasto en educación. No es suficiente la provisión de textos escolares, las pruebas de rendimiento académico son bajas —inferiores a las de muchos países asiáticos-, el día escolar es de corta duración (menos de tres horas y media en muchas escuelas urbanas), los días del calendario escolar (120) no corresponden a los del calendario oficial (180) y no tienen sino 4 o 5 horas $^{4}$. Los promedios de repitencia en los primeros años dan cuenta de la baja calidad de la enseñanza, lo mismo que los altos índices de deserción. Todo ello influye en la vinculación prematura al trabajo de los niños y niñas en edad escolar.

Un 20\% de los niños en edad escolar ingresa tardíamente, en relación con la edad establecida en cada país. La deserción se produce en general a partir de los 13 años y se acentúa a partir de los 14, edad en que se inicia en muchos países la inserción laboral legal (ibid). En los países con población indígena alta, como Ecuador, Guatemala y Perú, la matrícula es menor en estos grupos. Las diferencias por género han disminuido.

En síntesis, la educación primaria en la región muestra signos de baja calidad y acentuadas disparidades, tanto entre los países como en su interior. Aunque han aumentado el acceso y la cobertura, hay altos porcentajes de repitencia, deserción y bajas tasas de finalización del ciclo primario (BID 1993). Existe información sobre los motivos para no matricular a los niños en primaria que indican factores relacionados con el mismo sistema escolar (costos, distancia de la escuela, falta de transporte, insuficiencia

\footnotetext{
${ }^{4}$ En los países europeos, este promedio alcanza los 220 días de 6 horas diarias. En Japón y en algunos de los países asiáticos de reciente industrialización, el año lectivo tiene un promedio de 220 días de 9 horas diarias.
} 
de cupos) como la principal causa para no inscribirlos. En segundo lugar se mencionan "razones económicas" o la necesidad del trabajo infantil. Si el costo de oportunidad de asistencia a la escuela es muy alto para el hogar en relación con sus ingresos, habrá mayor vinculación al trabajo infantil.

En Guatemala, una encuesta realizada en 1994 con 600 niños que habían abandonado la escuela, mostró como motivos principales para esa decisión los relacionados con aspectos económicos y con las carencias de la escuela (profesores irresponsables, insuficiencia de recursos, inutilidad de las enseñanzas (40\%); los económicos (28\%) y el tener que trabajar (24\%). Es decir que la causa principal de la deserción escolar reside en la necesidad del trabajo. En los estudios sobre el trabajo infantil se ha subestimado la fuerza de las razones económicas no tanto en la contribución al hogar sino por los costos más altos de la escuela, y en términos del valor asignado al uso alternativo del tiempo del niño, aun no remunerado.

Por género, son más los varones que no se matriculan por razones de trabajo. Las jóvenes mayores de 15 años no se matricularon por tener que desempeñar los oficios domésticos. En el Ecuador uno de cada dos niños no se matriculó en la escuela por el costo de la educación o por el trabajo. Pero sería importante indagar más sobre las valoraciones de los padres acerca de la educación, y conocer el peso que tiene la mala calidad de la escuela y el bajo rendimiento escolar en determinar estas motivaciones, ya que ellas aparecen como determinantes del trabajo infanto-juvenil en todos los países de la región.

Para Colombia, en las 7 ciudades principales, las limitaciones de la escuela se constituyen en la principal causa de deserción, según respuestas de los mismos niños trabajadores, mientras que el trabajo es mencionado sólo por un $2 \%$ de ellos. Pero la deserción, en secundaria, si se debe a la necesidad de trabajar para el $21 \%$ de los varones y para el $11 \%$ de las mujeres, aunque se señala que el $40 \%$ de los hombres y el $28 \%$ de las mujeres no quisieron seguir estudiando, cifras que pueden explicarse de nuevo por la mala calidad de la escuela secundaria (Turbay y Acuña, 1995) ${ }^{5}$.

Los estudios de caso y diferentes evaluaciones del Banco Mundial y otras entidades destacan la baja calidad de la escuela como el principal problema del sistema escolar, junto con la inadecuada capacidad de gestión, su baja eficiencia, el acceso desigual a los servicios de educación y los recursos insuficientes de las escuelas.

Como consecuencia de estas deficiencias en los sistemas de educación, los trabajadores latinoamericanos registran un promedio de 5.2 años de escolarización, inferior en 2 o más años a la meta de universalización de la educación básica. La baja calidad de la educación: la región tiene la más alta tasa de repitencia del mundo, el número más bajo de horas anuales de escuela, y resultados muy deficientes en las pruebas de matemáticas y lenguaje (apenas comparables con los del África) (Londoño, 1995), lo cual conduce a que sólo una minoría de la población pueda educarse bien, acceder a empleos satisfactorios y tener condiciones de vida dignas.

Las investigaciones coinciden en señalar que si los niños y adolescentes tuviesen mayor acceso a la escuela, los resultados serían mejores oportunidades de empleo,

\footnotetext{
${ }^{5}$ Formas indirectas para medir los aspectos anteriores son los índices de repitencia, deserción y promoción. En Perú, 18 de cada 100 niños repiten algún curso de primaria, y un poco más del 30\% de los niños repiten el primer grado; en Guatemala y Brasil este porcentaje sube a casi 55. (Cifras de Unesco, citadas en Alarcón, 1996).
} 
aumento de su capacidad productiva y de su calidad de vida, acceso a la movilidad social y a la igualdad.

Políticas de ajuste estructural llevan a la privatización de servicios estatales y a la reducción del gasto social, lo que influye en el alza del costo de la educación para las familias que tienen que pagar matrículas, uniformes, transporte y útiles escolares. La presión de tales medidas recae principalmente sobre las mujeres jefes de hogar y sus niños, que se ven obligados a trabajar para asumir esos costos.

Para el Ecuador se conoce que el presupuesto para la educación ha disminuido constantemente desde 1980. El $90 \%$ de este presupuesto se emplea en pago de maestros, y apenas el $10 \%$ en desarrollo y mantenimiento de la infraestructura, capacitación, investigación pedagógica, etc. En Guatemala, durante los últimos 20 años el presupuesto destinado al sistema educativo no ha pasado nunca del $2 \%$ del PIB. El presupuesto de funcionamiento absorbe entre el 90 y el $97 \%$ del total de esos recursos. En Chile, el gasto público en educación representa (en 1988) el 3.6\% del PIB, mientras que en Brasil esa proporción es del 3.7\% y en Perú, del 3.4\%.

En particular en zonas rurales el quiebre entre el calendario agrícola y el calendario escolar es un elemento fundamental que provoca el abandono temporal $y$, en consecuencia, el atraso de estos educandos. Por las actividades laborales que desempeñan durante largas jornadas, estos niños llegan tarde a las aulas; en otros casos llegan cansados y pierden la concentración, o no tienen tiempo para estudiar y hacer las tareas en casa. En pocas palabras, trabajar significa una carga que daña un probable mejor desempeño en la escuela. La forma como el trabajo afecta el rendimiento en las aulas está asociada a la extensión de la jornada laboral, edad y tipo de actividad.

Hay una relación excluyente entre la actividad laboral y la asistencia escolar, como se demuestra en las investigaciones que sustentan esta publicación: el promedio de matriculados en Ecuador es mucho mayor entre los niños inactivos laboralmente (89\%) que entre los activos (58\%); la diferencia aumenta con la edad. En Colombia, los varones que trabajan sin asistir a la escuela, de 12 a 14 años, constituyen el $15.3 \%$ de ese grupo etario (mujeres: 6\%), mientras que en el grupo de 15 a 17 años, aumentan al 36.5\% los varones y al $12.8 \%$ las mujeres. Las proporciones de niños colombianos que estudian y trabajan a la vez son bajas (los varones de 12 a 14, el 6.2\%, las niñas del mismo grupo etano $1.9 \%$ ) y la diferencia por área indica que más niños rurales que urbanos pueden estudiar y trabajar a la vez. Pero en otros países la situación es diferente: por ejemplo, más de la mitad de los niños trabajadores en Brasil logran trabajar y estudiar a la vez.

La CEPAL advierte para la región que entre los 13 y 17 años de edad, los varones que trabajan tienen 1 a 2 años menos de educación que los que no trabajan, mientras que entre las niñas y adolescentes las diferencias más frecuentes se ubican entre 0.5 y 1.5 años de estudio. Estos niños y adolescentes terminarán acumulando un déficit educacional superior a 2 años de estudio con respecto a los que ingresarán al mundo laboral entre los 18 y 24 años. Dos años menos de educación significan un 20\% menos de ingresos mensuales durante la vida activa (CEPAL, 1995: 51).

\section{Políticas}

No cabe duda sobre la necesidad de modificaciones estructurales en las políticas estatales, particularmente en las sociales y en las dirigidas a la población infantil y juvenil. Hay que aplicar políticas que respondan a un modelo de crecimiento equitativo, tales 
como las enfocadas a generar empleos bien remunerados para adultos, aumentar el nivel adquisitivo de los salarios, crear recursos para invertirlos en particular en la educación y en la protección del medio ambiente para las generaciones futuras.

Hay un consenso creciente acerca de los principales objetivos de intervención en el área del trabajo infanto-juvenil, tales como los siguientes:

I. Erradicación progresiva del trabajo infantil para todos los niños y niñas menores de 12 años (o menores de 14, según las leyes nacionales), lo que sólo podrá lograrse con una lucha permanente, firme, creativa y vigorosa, de todos los sectores sociales, incluyendo la voluntad política del Estado.

II. Erradicación de los trabajos peligrosos, insalubres, nocivos o penosos para todos los niños, niñas y adolescentes.

III. Cumplimiento de las normas legislativas referidas al trabajo de adolescentes y a sus derechos laborales.

IV. Establecimiento de una política pública y popular de formación profesional para los adolescentes de 12 a 14 años, y la creación de empleos para los que hayan terminado el ciclo básico escolar. Talvez en este caso puedan aceptarse trabajos ligeros, de cortas jornadas laborales, que no interrumpan el proceso escolar. Como lo dicen Himes et al. (1994): "Para ambos grupos, los niños de 12 años y menos como también los de 13 y14 años, la centralidad de la escuela formal necesita ser el foco primario de las políticas y las intervenciones. Para el grupo de mayor edad (13-14), la educación vocacional y la profesional deben volverse opciones más viables para los estudiantes así inclinados, pero con un énfasis en la adquisición de habilidades para aprender y razonar, en vez de 'entrenamientos para el trabajo' (que mayormente ha fracasado en las escuelas). Es necesario tomar medidas como son ayuda financiera, salud y servicios de nutrición, convenios de trabajo/estudio, aprendizajes, apoyo especial para los que abandonan la escuela y otras, para permitir a los niños de familias en desventaja beneficiarse de las oportunidades de una educación apropiada tanto básica como también profesional/vocacional, de calidad aceptable, al menos hasta los 14 años."

V. Formulación y ejecución de políticas de protección legal y de profesionalización para los adolescentes de 14 a 18 años no cumplidos. De nuevo citando el texto anterior: "Para los jóvenes en el grupo de 15-18 años, que pueden legalmente trabajar en casi todos los países de la región -y a menudo deben trabajar, tanto por motivo de su propia educación como también para asistir a sus familias (incluyendo un creciente número de madres adolescentes o jóvenes, solteras), el énfasis primario debe cambiar hacia apropiados beneficios ocupacionales y protección para estos jóvenes trabajadores, incluso de ocupaciones peligrosas (que son ilegales para jóvenes en la mayoría de los países de la región). De cualquier modo, medidas complementarias en la educación, incluyendo aprendizajes y educación no formal para jóvenes adultos', pueden ayudar a garantizar que los trabajadores en este grupo de edades se beneficien de oportunidades más profesionales, orientadas al empleo y que 'expandan las opciones', que usualmente hay disponibles para jóvenes de familias de bajos ingresos y en otras formas de desventaja en América Latina y el Caribe. A medida que la gente joven pasa por sus años adolescentes, la necesidad de encontrar caminos más viables y apropiados para combinar educación con empleo se vuelve cada vez más importante para su futuro." 
VI. Definición de políticas socioeconómicas dirigidas a erradicar la pobreza; y de políticas sociales y salariales que favorezcan la democratización del acceso al ingreso, y la recuperación y mejoramiento del salario mínimo

VII. Movilización y organización de los adolescentes y jóvenes trabajadores en defensa de sus propios derechos en el trabajo y de sus derechos ciudadanos.

VIII. Fiscalización y control social y estatal eficientes, con presión nacional e internacional.

América Latina podría alcanza nueve años de educación para el conjunto de su fuerza de trabajo en menos de dos décadas con un esfuerzo financiero posible, según los estudios citados. La adopción de una senda dinámica de acumulación de capital humano, que representa un enorme esfuerzo de ahorro de las familias, cuando se acompaña de políticas globales que estimulen el crecimiento y la innovación en el conjunto de la economía, podría acelerar substancialmente el crecimiento económico, eliminar totalmente el exceso de desigualdad del continente y mejorar el nivel de vida del $70 \%$ de los latinoamericanos pobres.

Puede verse con mayor claridad que existe un vinculo muy fuerte entre el trabajo infantil y la educación primaria, como ya se venia documentando. En particular con los niños menores de doce años, debemos crear los mecanismos institucionales y sociales necesarios para que ellos dejen de trabajar en el corto plazo. Buena parte de esos niños y niñas acompañan a sus padres al trabajo sin hacer una contribución económica relevante para la subsistencia de la familia. En la mayoría de casos no existe justificación estrictamente monetaria de su presencia como trabajadores. Es necesario que los padres se convenzan de que es útil promover el alejamiento de estos niños del trabajo y paralelamente fortalecer su presencia y mejorar sus resultados en la escuela. En este campo deben hacerse las coordinaciones y presiones necesarias para que la educación a estos niños sea realmente gratuita. Para las familias urge recuperar la credibilidad en la escuela pública como espacio de instrucción y socialización.

Estos son los retos que tienen nuestros países en este campo. La Convención nos ha ayudado a verlos con mayor claridad. Este Seminario nos ayudará a profundizar en las maneras como todos tenemos que responder para alcanzar las metas que se desprenden de este crucial documento para el futuro de los niños y niñas de nuestra región, en especial los procesos relacionados con la erradicación de los trabajos peligrosos para todos los menores de 18 años, y de todos los trabajos de los niños menores de 12 años que impiden su escolarización y su desarrollo pleno.

\section{Referencias bibliográficas}

Alarcón Glasinovich, W., 1996. Trabajo y educación de niños y adolescentes en el Perú. UNICEF, Saritafé de Bogotá.

BID., 1993. Mejoramiento de la calidad de educación primaria en América Latina y el Caribe: Hacia el siglo XXI. Consultores: L. Wolff, E. Schiefelbein y J. Valenzuela. Informe no. 28. Programa de estudios regionales. América Latina y el Caribe. Dpto. Técnico.

CEPAL, 1995. Panorama social de América Latina. Edición 1995. Naciones Unidas, Santiago de Chile. 
Flórez, C.E., F. Knaul y R. Méndez, 1995. Niños y jóvenes: ¿cuántos y dónde trabajan? Ministerio de Trabajo y Seguridad Social, CEDE, Universidad de los Andes, Santafé de Bogotá.

García Méndez, E. y H. Araldsen, 1994. El debate actual sobre el trabajo infanto-juvenil en América Latina y el Caribe: tendencias y perspectivas. UNICEF, Santafé de Bogotá.

García Moreno, M., 1996. El trabajo y la educación de los niños y de los adolescentes en el Ecuador. UNICEF, Santafé de Bogotá.

IEE. Instituto de Estudos Especiais da PUC/SP., 1993. Trabalho do adolescente. Mitos e dilemas. Sáo Paulo.

Instituto Ser de Investigación, 1992. Evaluación del plan de universalización de la educación básica primada. Santafé de Bogotá.

Londoño, J.L., 1995. Pobreza, desigualdad, política social y democracia. Banco Mundial. Departamento Técnico de América Latina.

Naciones Unidas, 1995. Estudio económico y social mundial. Nueva York.

Petras, J., 1995. "La recuperación económica de América Latina, el mito y la realidad, en Nueva Sociedad (Caracas), 137: pp. 164-179.

Rizzini, I., I. Rizzini y F. R. Borges, La fuerza de la infancia no está en el trabajo. UNICEF, Santafé de Bogotá.

Rodrigues dos Santos, B., 1996. Trabalho infantil no Brasil: Um estudo das estratégias e políticas para sua eliminaçáo. DNI-ISPCAN-IWGCL, SáoPaulo.

Rodríguez L., C.A., 1996. Guatemala: el trabajo y la educación de los niños, niñas y adolescentes. UNICEF, Santafé de Bogotá.

Salazar, M.C. y M. Gárate, 1996. Informe sobre trabajo infantil en América Latina. International Working Group on Child Labour/Defence for Children International. Amsterdam.

Turbay Restrepo, C. y E. Acuña Vargas, 1996. Trabajo infanto/juvenil y educación básica en Colombia. UNICEF, Santafé de Bogotá.

UNICEF, 1992 . Latin Ameríca Special Report 5, 10: pp. 2-3. 1992a. Los niños de las Américas. UNICEF, Santafé de Bogotá.

Vélez, E., E. Schiefelbein y J. Valenzuela, 1994. Factores que afectan el rendimiento académico en la educación primaria: revisión de la literatura para América Latina y el Caribe, Banco Mundial. 